\title{
Protocols protocol and standard operating procedures
}

Experiences have an important role in medicine. Everything cannot be covered by the avilable medical books. In the early days of medical science, most of the medical texts covered recommendation of senior doctors emanating from years of experience gained by observation of diseases and the treatments administered in patient care. Though some of those annecdotal observations were later proven wrong, most of the medical knowledge that we have today is built upon such observations. A simple observation by Ignaz Semmelweiss in 1840s, an assistant in the obstetrics department of the General Hospital in Vienna, Austria that death rate of women by puerperal fever was higher in the ward attended by medical students after their visit to postmortem room led to the development of the concept of germs and wound infection. ${ }^{1}$ But there are many more ideas that were the norms of their times which were disregard in due course. As we enter the 21st centurary, the days of 'ín my experince ...' are history. This is an era of evidence based medicine (EBM), and this is what we should adopt to optimize our patient management.

The practice of medicine has been compared to music. ${ }^{2}$ The new archetype of evidence based medicine and the notion of clinical experience relates to technical skill and individual artistry. EBM is "the conscientious, explicit and judicious use of current best evidence in making decisions about the care of the individual patient. It means integrating individual clinical expertise with the best available external clinical evidence from systematic research."3 Some might consider EBM as rigid, unstructured and uninspiring like a poorly performed classic, while academics consider clinical experience as unpredictable likening it to punk rock: uncontrollable and chaotic. However, the best practice is akin to a good jazz, combining technical mastery with personal improvisation.

Though, bringing EBM to practice requires some effort, it is not that difficult. In simple terms, when we diagnose wound infection, we should now know the criteria of surgical site infection. Especially, in medical training, all medical students should be well versed in the standard operating procedures (SOPs), which have been tried and tested in research or in the department. If all the students tried their own methods, they might be lucky once or twice, but ultimatly it will lead to a rise in complications.

Protocols have been encouraged in patient management to provide a standard of care. It not only improves data collection, efficiency and quality of life but also leads to reduction of cost and human error that we might commit. When things are defined, it helps to administer a uniform level of care to all the patients. Data keeping also improves paving way for further research. It is more important in medical institutions where new residents can provide patient care with less risk of errors.

Thus, departments should have their own protocols and Standard Operating Procedures, so that everybody should learn the same good standards. Once the basic SOP is mastered, then only can one add their own touch after seeing many operations from various experts. In this age of competetive knowledge and skill assessment, it is not the question of protocol/SOP vs experience, it is a question of making a competent doctors who know their basics from the department's protocol/SOP and has learned much from their seniors experiences. The aim is not only to make good specialist surgeons, physicians, but to make a good doctor who looks after the whole patient and not only one's specialty organ.

Even protocols cannot be rigid and need to change with time. Hence, they are subjected to reviews and analysis. There was a time when operations used to be performed in a theatre hence the term 'operation theatre' but since the knowledge of infection and asepsis, various stringent measures are undertaken to maintain sterility at the operation theatre these days. Protocals need to be dynamic with constant overviews and revisions with time. What is considered a protocol today might well be outdated tomorrow. Only time will unravel the mystery of human body to a full extent, so for present it is for us to make and follow protocols.

\section{References}

1. Youngson AJ. The scientific revolution in Victorian medicine. London: Croom Helm; 1979, 2371979.

2. Shaughnessy AF, Slawson DC, Becker L. Clinical jazz: harmonizing clinical experience and evidence-based medicine. J Fam Pract 1998; 47:425-8.

3. Sackett DL, Rosenberg WM, Gray JA, Haynes RB, Richardson WS. Evidence based medicine: what it is and what it isn't [Editorial]. BMJ 1996;312:71-2. [PMID: 8555924] 